【国際セッション】

\title{
Decreased motor unit firing rate and force control in older men
}

\section{Kallio Jouni', Linnamo Vesa', Avela Janne', Kanervo Mika', Segaard Karen', Komi Paavo'}

'Neuromuscular Research Center, Department of Biology of Physical Activity, University of Jyvaskyla, Finland

${ }^{2}$ National Institute of Oocupational Health, Copenhagen, Denmark

\section{Introduction}

Ageing is related to muscle atrophy that leads to decreases in muscle force. The largest changes are found in fast muscle fibres and fast force production, reducing the capability to recover from sudden balance disturbances. Also ageing-related decresse in force control has been found, as indicated by an increase in force fluctuations and motor unit (MU) firing variability (Galganski et al. 1993). Possibly due to differences in measurement protocols and muscles, the results concerning the effects of ageing on motor unit firing rate are, however, scmewhat contradictory (for review. $5 e$ Roos et al. 1997). The purpose of the present study was to investigate the age-related changes in the firing properties of the Soleus muscle, which has an important fole in locomotion and balance.

\section{Methods}

8 YOUNG $(27.1+3.2 \mathrm{yr} .177 .1+7.8 \mathrm{~cm} 71.7+4.7 \mathrm{~kg})$ and 9 OLD $(70.2+4.1 \mathrm{yr}, 176.3+4.5 \mathrm{~cm} .84 .9+10.7 \mathrm{~kg})$ physically active males served as subjects in the study. The subjects performed plantar fiexions while seated in an ergometer. The trials consisted of isometric ramp contractions at a very slow rate (1\%/MVC/s) and level contractions at 10 and 20\%MVC. EMG activity of Soleus (SOL) was measured with a bipolar surface electrode. For motor unit (MU) identification four novel bipolar fine-wire electrodes were inserted into SOL. Signal decomposition, motor unit identification and date analysis were performed with a special Daisy-software (Olsen et al. 2001). From the motor unit data, firing rates (FR) were calculated at recruitment threshold (TH, see Fig. 1.) and at two submaximal EMG activity levels (L10 \& L20\%EMG-max). For analysis, motor units were divided into two groups (TH10\% and TH20\%) according to the activation level. The variabilites in FR and force were determined by coefficient of variation (FR CV\%: SD of instantaneous FR/mean FR and F CV\%: SD of force curve/mean force, respectively).

\section{Results}

The maximal voluntary plantarflexion force was $21.4 \%$ lower in OLD as compared to YOUNG ( $P=0.03$ ). Also FR was significantly lower in OLD at TH10\%. TH20\% and L10\%. The difference at L20\% did not resch statistical difference (Fig.2). In OLD force fuctuation was largor at L10\%, which was accompanied with a slight increase [n.s] in FR variation (Fig 3)

\section{Discussion/Conclusion}

FR was lower in OLD at recruitment threshold and in $\mathrm{L} 10 \%$, which is in line with earlier findings in other distal muscles, e.g. Tibislis Antericr (Comelly et al 1999) and Adductor Digiti Minimi (Nelson at al. 1984) Also the larger F CV $\%$ in OLD at L1O\% was in line with previous studies with smaller hand muscles (Gelganski et al. 1993). These findings suggest that the age-related changes in motor unit control do exist and they can be messured a'so in large leg extensors that play an important role in human locamotion and balance control

Fig. 1. Identification of three motor units. Inses: For MU identification, raw date (bottom) and two different filtered signals were used

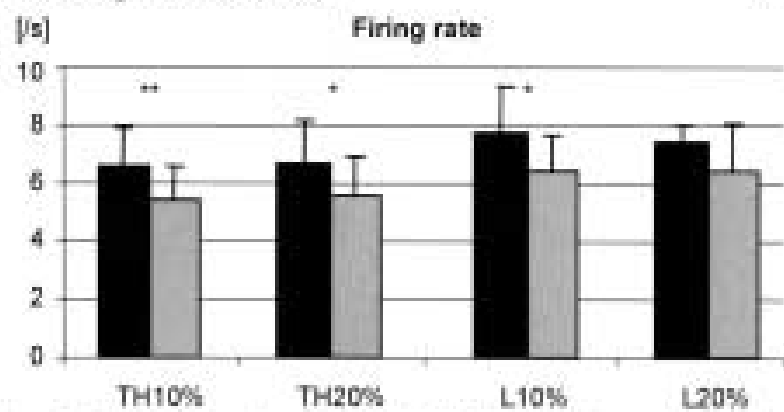

Fig. 2: Firing rates $(x+S D)$ of YOUNG (black) and OLD (gray) at threshold (TH) and in level contractions ( $\mathrm{L}$ ).

\section{References}

Roos et al. (1997) Murcie Nerve Vol 22: 1094-1103

Galganski of al (1993) J Neurophys. Vol 69: 2108-15

Connelly ef al (1999) J.App. Physiol Vol 87; 843-852
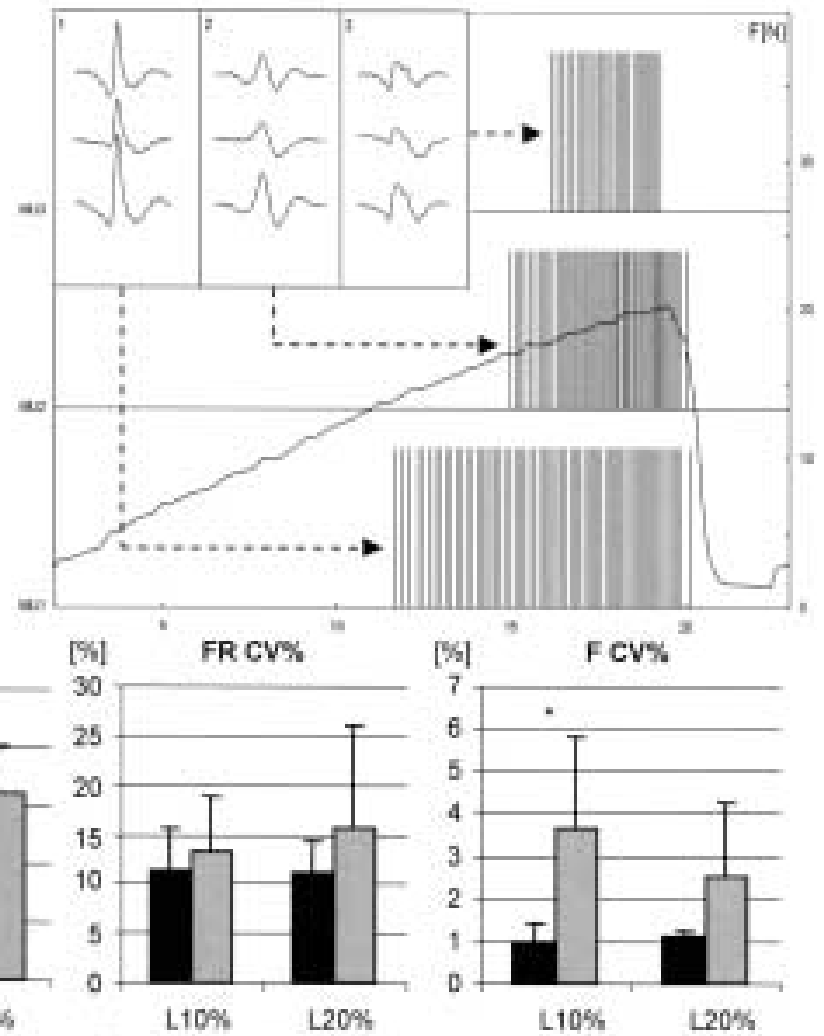

Fig. 3: Coefficient of variation $(x+S D)$ of firing rate $(F R)$ and force $(F)$ in level contractions 